\title{
A Unified Pedestrian Routing Model Combining Multiple Graph-Based Navigation Methods
}

\author{
Peter M. Kielar, Daniel H. Biedermann, Angelika Kneidl, André Borrmann
}

\begin{abstract}
The navigation behavior of pedestrians in street networks can be forecast by computer simulations based on routing models. These models characterize pedestrians' route choices regarding a variety of factors. However, the spatial cognition aspects are often omitted in routing models; thus, the diversity of predictable routes is limited. Here, we present a unified routing model that describes route choices of pedestrians by integrating the spatial cognitive aspects of allocentricbased and egocentric-based navigation. We achieved this by combining graph-based routing methods, each formalizing a single spatial cognitive aspect. In addition, we present a generic calibration method for our model. For validation, we show that our model is able to correctly predict the routing behavior of pedestrians in a case study.
\end{abstract}

\section{Introduction}

Every day, pedestrian navigate and walk in the street networks of cities. It is possible to forecast the pedestrians' navigational behavior and predict the chosen routes by means of computer simulations. However, individual human navigation is influenced by many different factors [10] and there is still no pedestrian routing behavior

Peter M. Kielar

Chair of Computational Modeling and Simulation, Technische Universität München Arcisstr. 21 D-80333 München e-mail: peter.kielar@tum.de

Daniel H. Biedermann

Chair of Computational Modeling and Simulation, Technische Universität München Arcisstr. 21 D-80333 München e-mail: daniel.biedermann@tum.de

Angelika Kneidl

accu:rate Institute for crowd simulation, Isarwinkel 14 D-81379 München e-mail: ak@accu-rate.de

André Borrmann

Chair of Computational Modeling and Simulation, Technische Universität München Arcisstr. 21 D-80333 München e-mail: andre.borrmann@tum.de 
model that can predict the complete richness of pedestrian route choices.

In our previous research, different aspects of human spatial cognitive abilities were integrated successfully, and four different graph-based pedestrian navigation algorithms were presented [8]. Nonetheless, each method depicts only a specific type of navigation behavior - but, as shown by research on spatial cognition, human navigational abilities are more fine grained [5, 10]. Here, we present a Unified Pedestrian Routing Model that combines these four routing methods in a single model. By applying our approach to a pedestrian simulation, one can simulate a more diverse range of pedestrian wayfinding behavior.

\subsection{Related work}

In contemporary research, there are several different approaches to model human navigation behavior. Graph-based concepts utilize routing algorithms to determine an ordered set of street network joints to describe the path a pedestrian takes [8, 7]. Graph-generation algorithms create the underlying routing network for the routing algorithms, based on the scenario geometry $[9,3]$. The corridor map method is a concept that uses a route-map graph as well as clearance information to construct a corridor covering the traversable scenario layout from an origin to a destination [4]. When the corridor is completed, a simulated pedestrian navigates inside its boundaries towards a given destination. Another concept is the floor field method and its derivates [1]. These methods compute distances on a grid that covers the simulation scenario as underlying data structure. Each grid cell is able to hold values - dynamically or statically - which are subject to gradual changes according to destination proximity and ground floor traversability.

\subsection{Spatial cognitive concepts}

The large field of research on spatial cognition contributes to improve our understanding of people's navigational abilities and limitations [10]. Human navigation abilities depend on the accuracy of perceiving spatial information, the competence to generate a spatial representation of the environment, as well as the efficiency of utilizing the spatial representations [10]. In general, spatial representations of the environment are denoted as cognitive maps. Thus, wayfinding abilities are directly related to the construction and processing of such maps [5].

Research on spatial cognitive abilities was able to prove that people use route-based, survey-based, or fuzzy intermediate strategies for navigation $[5,10]$. The routebased navigation describes that a sequence of egocentric actions has to be carried out to recreate a route. Hence, little overall knowledge about the environment and relation between locations is needed. In contrary, the survey-based navigation approach is an allocentric strategy. People who use this strategy have a general understanding 


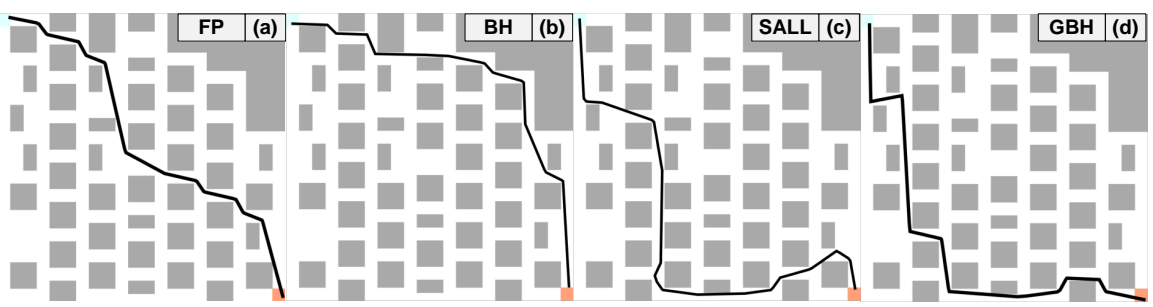

Fig. 1 (a) - (d): The routes found by the four different basic routing models in a pedestrian simulation. The pedestrians started at the top-left corners and walked to the corresponding bottom-right destination. The graph-generation method of [9] was used for this artificial scenario.

of relations and distances concerning a certain area, so they are able to find new direct paths from their current location to a not directly visible destination.

However, people do not simply choose one of these two strategies, but generate intermediate and mixed routing solutions based on the integrity of their cognitive map, the reliability of their memory retrieval processes, their abilities to apply the information into actions, and the navigation task at hand [10, 5]. This predicts that intermediate variants of navigation methods must exist. The proposed Unified Pedestrian Routing Model captures these intermediates variants of route-based and survey-based navigation behavior in a single wayfinding model.

\section{Graph-based navigation methods}

Graph-based routing methods for pedestrians predict walking paths from an origin to a destination either iteratively or directly in an algorithmic manner. The distinction is based on the egocentric routing strategy (iteratively) and the allocentric routing strategy (directly) outlined in Section 1.2. Direct routing solves the routing problem by providing an optimal path to the destination, based on shortest path solutions $[2,6]$. In contrast, iterative routing algorithms provide the next vertex to visit stepwise, based on local optima. Previous work [8] includes the development of two direct routing methods - the Fastest Path (FP) and the Beeline Heuristics (BH) - as well as two iterative routing methods - the Greedy Beeline Heuristics (GBH) and the Straight and Long Legs (SALL). Figure 1 presents example routes generated by a pedestrian simulation that applies these methods on an artificial scenario.

\subsection{Direct routing methods}

We build upon two direct routing methods, the Fastest Path (FP) and Beeline Heuristics (BH) algorithms [8]. Both methods model the routing behavior of pedestrians who have a profound knowledge of the street network. 
The FP method is based on Dijkstra's algorithm [2]. The weight calculation method of the FP combines the distance $d_{i j}$ between current vertex $i$ and a successor vertex $j$ as well as a velocity $v_{i j}$, which is based on the number of pedestrians $p_{i j}$ walking alongside a directed edge $e_{i j}$. If an edge $e_{i j}$ is not visible to a pedestrian, a standardized mean velocity $v_{m}$ is used.

$$
w_{j}=w_{i}+d_{i j} / v_{i j}
$$

Because the FP algorithm has to be applied each time a pedestrian visits a vertex, the edge weights $v_{i j}$ are updated corresponding to the velocities of the pedestrians $v_{x i j}$ on an edge $e_{i j}$. If the number of pedestrians $p_{i j}$ at an edge is zero, a standardized mean velocity $v_{m}$ is used for $v_{i j}$.

$$
v_{i j}=\left(\sum_{x=1}^{k} v_{x i j}\right) / p_{i j}
$$

The $\mathrm{BH}$ algorithm is based on the A-Star algorithm [6] and describes the tendency to follow the beeline towards a destination. The algorithm of the weight calculation of the $\mathrm{BH}$ routing method integrates the beeline distance $d_{j g}$ from the next vertex $j$ to the destination vertex $g$, the distance $d_{i j}$ between current vertex $i$ and a successor vertex $j$, and a constant $\beta$. We omitted the random distance estimation error presented in [8]. Nonetheless, we kept the constant factor $\beta=1.5$ of [8] to strengthen the beeline part of the calculation.

$$
w_{j}=w_{i}+d_{i j}+d_{j g} \cdot \beta
$$

The BH method's weight update uses the Dijkstra's Algorithm update procedure.

\subsection{Iterative routing methods}

The Greedy Beeline Heuristics (GBH) and the Straight and Long Leg methods (SALL) are the iterative routing concepts we build up upon [8]. They model the routing behavior of pedestrians who are not familiar with the street network.

The SALL method models the tendency to walk alongside straight and long streets. The route choice is based on the angle $\gamma_{a i j} \in[0, \pi]$ in between the last vertex $a$ and a next vertex $j$, as well as the relative distance reduction $d_{j h g} / d_{j g}$ to the destination vertex $g$. The value $d_{j h g}$ is calculated based on the vertex $h$, which is the farthest vertex within the projection of an arc of the angle of $\pm \mu$. The arc is centered alongside $e_{i j}$ and its successor edges in succession. We set $\mu$ to $\pi / 12$ for scenarios with rectangular street networks, and $\pi / 9$ otherwise. For the calculation of the weight of a successor vertex $j$, the angle $\gamma_{a i j}$ improves the weight in the case of straight roads. The successor vertex with the smallest result is selected as the next vertex to visit.

$$
w_{j}=(1-\alpha) \cdot\left(d_{j h g} / d_{i g}\right)+\alpha \cdot\left(1-\left|\gamma_{a i j}\right| / 180^{\circ}\right)
$$


The constant $\alpha=0.75$ increases the influence of the angle parameter between zero and one. The value for $\alpha$ was determined by multiple preemptive test simulations. The GBH describes a BH approach for pedestrians who are not familiar with the street network. Therefore, a simulated pedestrian can get lost by greedily following the beeline to the destination. For the route choice, the vertex with the smallest weight $w_{i j}$ is selected as the next vertex to visit. The weight is influenced by the beeline distance $d_{j g}$ from a successor vertex $j$ to destination $g$ only.

$$
w_{j}=d_{j g}
$$

\section{The Unified Pedestrian Routing Model}

The Unified Pedestrian Routing Model (UPRM) integrates all routing methods described in Section 2. By doing so, the integration is not a mutual exclusive application of routing algorithms, but a calculation-based integrative concept.

\subsection{Merging the graph-based methods}

The UPRM is an iterative routing method to model the route-choice decision behavior of selecting the next vertex to visit at a street junction, based on the four base algorithms. The weight calculation for UPRM is:

$$
w_{j}=\zeta \cdot w_{j}^{F P}+\eta \cdot w_{j}^{B H}+\kappa \cdot w_{j}^{G B H} / \max \left(w_{j}^{G B H}\right)+\psi \cdot w_{j}^{S A L L} / \max \left(w_{j}^{S A L L}\right)
$$

The successor vertex with the smallest weight $w_{j}$ of the current vertex $i$ will be selected as the next vertex to visit, as for the previous iterative methods.

The UPRM integrates the Greedy Beeline Heuristics (GBH) and Straight and Long Legs (SALL) methods by assessing the weights of all adjacent vertices $j$ of vertex $i$. These weights are normalized by the maximal weight of all successor vertices of vertex $i$ for each method separately. The Fastest Path (FP) and the Greedy Beeline (GB) algorithms contribute temporary calculations of the optimal paths, starting from vertex $i$. The first vertex of each optimal path is compared to the adjacent vertices $j$ of the current vertex $i$. If a successor vertex of $i$ is part of the optimal path, the weight $w_{j}^{F P}$ (or respectively $w_{j}^{B H}$ ) is zero - and otherwise, it is one.

The resulting weights for each of the original methods are additively combined based on influence factors. These factors describe how strong a pedestrian is associated to a routing strategy. The factors $\zeta$ and $\eta$ describe a high familiarity with the route network, whereas the factor $\kappa$ and $\psi$ describe a lesser knowledge of the network. The values range in $[0,1]$, but a single factor with a value larger than zero is mandatory. Hence, the factor concept also models interfering knowledge and uncertainty due to overlapping or underrepresented factor combinations. 
Table 1 Factor combinations derived from the calibration method for an artificial scenario.

\begin{tabular}{r|rrrrrrrrrrrrrrrrrr} 
& $\mathrm{a}$ & $\mathrm{b}$ & $\mathrm{c}$ & $\mathrm{d}$ & $\mathrm{e}$ & $\mathrm{f}$ & $\mathrm{g}$ & $\mathrm{h}$ & $\mathrm{i}$ & $\mathrm{j}$ & $\mathrm{k}$ & $\mathrm{l}$ & $\mathrm{m}$ & $\mathrm{n}$ & $\mathrm{o}$ & $\mathrm{p}$ & $\mathrm{q}$ & $\mathrm{r}$ \\
\hline$\zeta$ & 0.00 & 0.26 & 0.12 & 0.10 & 0.06 & 0.12 & 0.20 & 0.00 & 0.18 & 0.21 & 0.05 & 0.08 & 0.00 & 0.07 & 0.05 & 0.31 & 0.24 & 0.00 \\
$\eta$ & 1.00 & 0.42 & 0.31 & 0.37 & 0.30 & 0.24 & 0.23 & 0.00 & 0.12 & 0.00 & 0.15 & 0.16 & 0.14 & 0.03 & 0.00 & 0.01 & 0.00 & 0.00 \\
$\kappa$ & 0.00 & 0.81 & 0.75 & 0.99 & 0.94 & 0.40 & 0.75 & 0.00 & 0.02 & 0.03 & 0.50 & 0.75 & 0.33 & 0.43 & 0.99 & 0.57 & 1.00 & 1.00 \\
$\psi$ & 0.00 & 0.78 & 0.45 & 0.02 & 0.15 & 0.87 & 0.71 & 1.00 & 0.75 & 0.76 & 0.62 & 0.95 & 0.91 & 0.68 & 0.67 & 0.73 & 0.69 & 0.00
\end{tabular}

\subsection{Model calibration}

The UPRM predicts the navigational paths based on the influence factors of the underlying routing algorithms. Therefore, a calibration method has to find influence factor combinations that yield realistic pedestrian routes for a scenario layout. We suggest a generic calibration approach that finds factors by a test simulation comprising a finite set of test pedestrians, initialized with random factors. We evaluated the calibration method by running a simulation with 1000 independent test pedestrians for an artificial world scenario. The simulated routes are compared by an extended version of the turning angle metrics [8]. The extended method removes redundant factor combinations that generate identical routes, based on the turning angles along the routing path. Hence, the method accepts only factor combinations that generate unique routes that reach the destination. Using this method, we found 18 feasible factor combinations for the artificial test scenario (See Table 1). Figure 2 shows the resulting routes based on the found factor combinations. Hence, our calibration method yields plausible and usable combinations for any layout.

\section{Model validation}

We applied the Unified Pedestrian Routing Model (UPRM) to a case study, an annual music festival, to validate the model by comparing the routes of real and simulated pedestrians. Thus, we captured the routing behavior of approximately 700 of the 5000 festival visitors in 71 GPS measurements. The visitors traveled from a public transport station to the venue on walkways and streets that were closed for car traffic. Figure 3 (a) - (f) shows the six different routes chosen by the visitors. For validation, we calibrated the model as described in Section 3.2 and simulated the travelers' route choices. As visualized in Figure $3(\mathrm{~g})$ - $(\mathrm{k})$, the simulation results reveal that the UPRM predicts six different routes and forecast the route choices of the routes A to E correctly. The model could not predict route F completely, but misses to forecast one single route choice only - compare Figure 3 (f) and (l). Naturally, the four original routing methods of [8] could predict the route choices of four routes correctly. In summary, the UPRM forecasts the route choices of pedestrian quite well, but seems to contain a minor flaw. We assume that the found uncertainty in predicting route $\mathrm{F}$ is based on a non-optimal calibration of the routing-graph generation algorithm. It might be necessary to include further graph generation methods. 


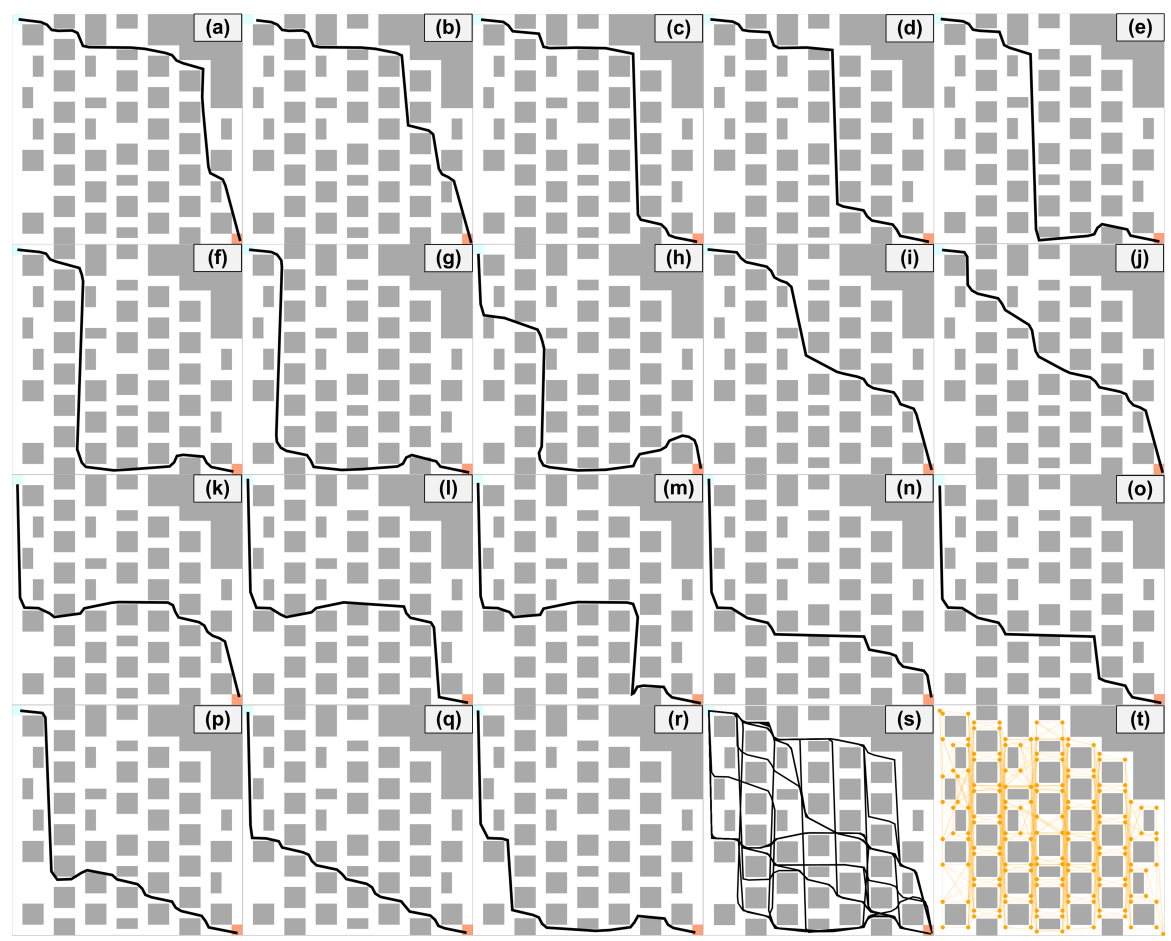

Fig. 2 (a) - (r): Routes created by the UPRM based on Table 1 for the artificial simulation scenario. All routes start at the top-left and end at the bottom-right. (s) shows all routes, and (t) represents the used underlying graph [9].

\section{Conclusion}

The Unified Pedestrian Routing Model (UPRM) represents a mathematical combination of pedestrian routing models from previous research. The model predicts route-based and survey-based navigation behavior and, more importantly, intermediate and mixed variants of these routing strategies. By utilizing pedestrian simulations, we showed that the UPRM is able to forecast a very large set of realistic navigation routes. Additionally, we validated the UPRM using a case study. We could forecast most of the pedestrian route choices of the study. Therefore, our approach of combining existing graph-based pedestrian routing methods to simulate more realistic routing behavior was successful. Nonetheless, further validation studies are in preparation. We also aim to integrate herding-behavior and a prediction of the relative frequency of route usage in further extensions of the UPRM.

Acknowledgements This work is supported by the Federal Ministry for Education and Research (Bundesministerium für Bildung und Forschung, BMBF), project MultikOSi, under grant FKZ 13N12823. We would like to thank our student assistants for data acquisition during the festival. 


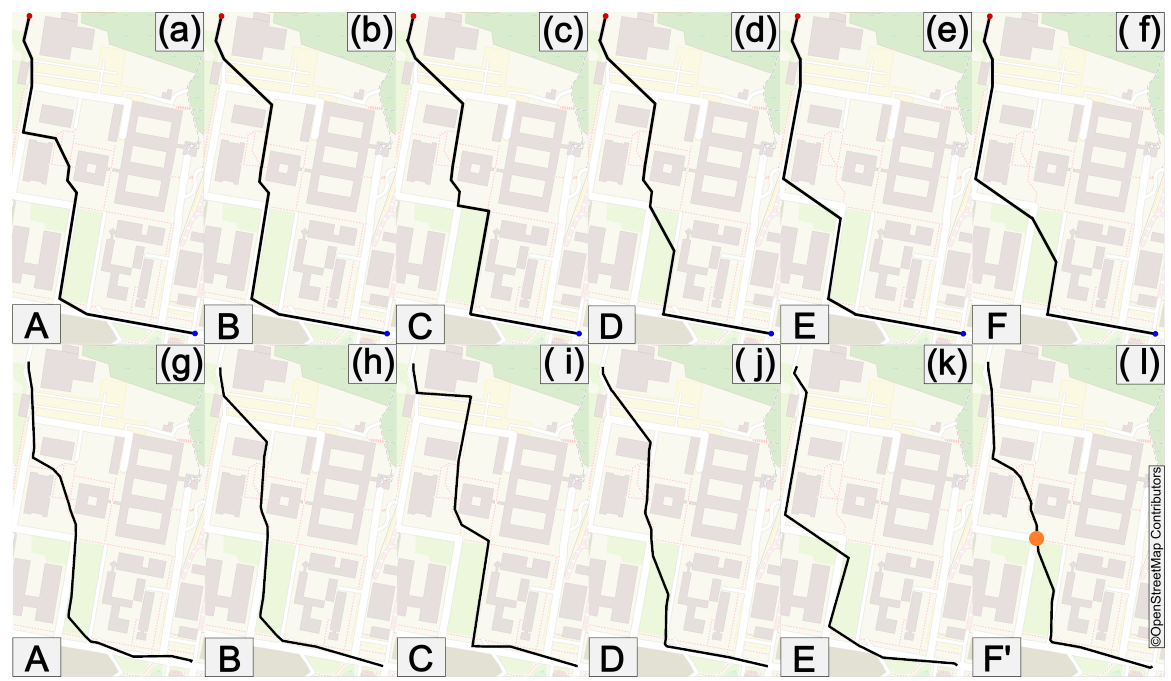

Fig. 3 (a) - (f): The measured walking routes of the festival visitors A to F.(g) - (l) show the simulation walking routes A to F'. The orange marker in (1) indicates the falsely predicted route choice. All routes start at the bottom and end at the top. An extended version of the graph-generation method of [9] was used for the case study scenario.

\section{References}

1. Burstedde, C., Klauck, K., Schadschneider, A., Zittartz, J.: Simulation of pedestrian dynamics using a two-dimensional cellular automaton. Physica A: Statistical Mechanics and its Applications 295(3-4), 507-525 (2001)

2. Dijkstra, E.W.: A note on two problems in connexion with graphs. Numerische Mathematik 1(1), 269-271 (1959)

3. Geraerts, R., Overmars, M.H.: Creating high-quality roadmaps for motion planning in virtual environments. In: 2006 IEEE/RSJ International Conference on Intelligent Robots and Systems, pp. 4355-4361 (2006)

4. Geraerts, R., Overmars, M.H.: The corridor map method: a general framework for real-time high-quality path planning. Computer Animation and Virtual Worlds 18(2), 107-119 (2007)

5. Golledge, R.G.: Human wayfinding and cognitive maps. Wayfinding behavior: Cognitive mapping and other spatial processes pp. 5-45 (1999)

6. Hart, P.E., Nilsson, N.J., Raphael, B.: A formal basis for the heuristic determination of minimum cost paths. IEEE Transactions on Systems Science and Cybernetics 4(2), 100-107 (1968)

7. Höcker, M., Berkhahn, V., Kneidl, A., Borrmann, A., Klein, W.: Graph-based approaches for simulating pedestrian dynamics in building models. eWork and eBusiness in Architecture, Engineering and Construction pp. 389-394 (2010)

8. Kneidl, A., Borrmann, A.: Methoden zur Abbildung menschlichen Navigationsverhaltens bei der Modellierung von Fußgängerströmen. Ph.D. thesis, Technical University Munich (2013)

9. Kneidl, A., Borrmann, A., Hartmann, D.: Generation and use of sparse navigation graphs for microscopic pedestrian simulation models. Advanced Engineering Informatics 26(4), 669$680(2012)$

10. Wolbers, T., Hegarty, M.: What determines our navigational abilities? Trends in Cognitive Sciences 14(3), 138-146 (2010) 